\title{
The smallest one-realization of a given set III
}

\author{
Kefeng Diao ${ }^{\mathrm{a}}$ Ping Zhao ${ }^{\mathrm{a}}$ Kaishun Wang ${ }^{\mathrm{b} *}$ \\ a. School of Science, Linyi University, Linyi, Shandong, 276005, China \\ b. Sch. Math. Sci. \& Lab. Math. Com. Sys., Beijing Normal University, Beijing 100875, China
}

\begin{abstract}
In [The smallest one-realization of a given set, Electronic J. Combin. 19 (2012), $\sharp \mathrm{P} 19]$, we determined the minimum number of vertices of one-realizations of a given finite set $S$, and constructed the corresponding mixed hypergraphs. In this paper, by finding some of their spanning sub-hypergraphs, we determine the minimum number of $\mathcal{D}$-deges (resp. $\mathcal{C}$-edges) of one-realizations of $S$. As a result, we partially solve an open problem proposed by Tuza and Voloshin in 2008.
\end{abstract}

Key words: hypergraph coloring; mixed hypergraph; feasible set; one-realization.

\section{Introduction}

A mixed hypergraph on a finite set $X$ is a triple $\mathcal{H}=(X, \mathcal{C}, \mathcal{D})$, where $\mathcal{C}$ and $\mathcal{D}$ are families of subsets of $X$, called the $\mathcal{C}$-edges and $\mathcal{D}$-edges, respectively. A bi-edge is an edge which is both a $\mathcal{C}$-edge and a $\mathcal{D}$-edge. If $\mathcal{C}=\mathcal{D}, \mathcal{H}$ is a bi-hypergraph. If each edge has $r$ vertices, then $\mathcal{H}$ is $r$-uniform. When $\mathcal{C}=\emptyset$ (resp. $\mathcal{D}=\emptyset), \mathcal{H}$ is a $\mathcal{D}$ hypergraph (resp. $\mathcal{C}$-hypergraph). A sub-hypergraph $\mathcal{H}^{\prime}=\left(X^{\prime}, \mathcal{C}^{\prime}, \mathcal{D}^{\prime}\right)$ of $\mathcal{H}=(X, \mathcal{C}, \mathcal{D})$ is a spanning sub-hypergraph if $X^{\prime}=X$, and $\mathcal{H}^{\prime}$ is a derived sub-hypergraph of $\mathcal{H}$ on $X^{\prime}$, denoted by $\mathcal{H}\left[X^{\prime}\right]$, when $\mathcal{C}^{\prime}=\left\{C \in \mathcal{C} \mid C \subseteq X^{\prime}\right\}$ and $\mathcal{D}^{\prime}=\left\{D \in \mathcal{D} \mid D \subseteq X^{\prime}\right\}$.

A proper $k$-coloring of $\mathcal{H}$ is a mapping from $X$ into a set of $k$ colors so that each $\mathcal{C}$-edge has two vertices with a Common color and each $\mathcal{D}$-edge has two vertices with Distinct colors. A strict $k$-coloring is a proper $k$-coloring using all of the $k$ colors, and a mixed hypergraph is $k$-colorable if it has a strict $k$-coloring. A coloring of $\mathcal{H}$ may be viewed as a partition of its vertex set, where the color classes are the sets of vertices assigned to the same color, so a strict $n$-coloring $c=\left\{C_{1}, C_{2}, \ldots, C_{n}\right\}$ of $\mathcal{H}$ means that $C_{1}, C_{2}, \ldots, C_{n}$ are the $n$ color classes under $c$. The set of all the values $k$ such that $\mathcal{H}$ has a strict $k$-coloring is called the feasible set of $\mathcal{H}$, denoted by $\mathcal{F}(\mathcal{H})$. For each $k \in \mathcal{F}(\mathcal{H})$, let $r_{k}$ denote the number of partitions of the vertex set. For a set $S$ of positive integers, we say that a mixed hypergraph $\mathcal{H}$ is a realization of $S$ if $\mathcal{F}(\mathcal{H})=S$. A mixed hypergraph $\mathcal{H}$ is a one-realization of $S$ if it is a realization of $S$ and $r_{k}=1$ for each $k \in S$. When one considers the colorings of a mixed hypergraph, it suffices to assume that each $\mathcal{C}$-edge has at least three vertices. The study of the colorings of mixed hypergraphs has made a lot of progress since its inception [9]. For more information, we would like refer readers to [5, 8, 10, 11].

Kündgen et al. [7] initiated a systematic study of planar mixed hypergraphs, and found a one-realization of $\{2,4\}$ on 6 vertices for planar hypergraphs. Bujtás and Tuza

\footnotetext{
${ }^{*}$ Corresponding author: wangks@bnu.edu.cn
} 
[1] gave a necessary and sufficient condition for a set to be the feasible set of an $r$ uniform mixed hypergraph. In [12], we introduced a new construction of hypergraphs, and characterized the feasible set and chromatic spectrum of a 3-uniform bi-hypergraph. Jiang et al. [4] proved that a set $S$ of positive integers is a feasible set of a mixed hypergraph if and only if $1 \notin S$ or $S$ is an interval, and determined the minimum number of vertices of realizations of $\{s, t\}$ with $2 \leq s \leq t-2$. Král [6] initiated the study of the minimum number of vertices of one-realizations of $S$, and obtained an upper bound. In [13], we determined this minimum number. We further determined the minimum number of vertices of 3-uniform bi-hypergraphs which are one-realizations of $S$ in [14].

Note that the feasible set of any $r$-uniform $\mathcal{C}$-hypergraph contains $\{1, \ldots, r-1\}$ as a subset. On $n$ vertices, the minimum number of $r$-element $\mathcal{C}$-edges to generate this smallest possible feasible set is $\lceil n(n-2) / 3\rceil$ in the particular case of $r=3$, but only some lower and upper estimates of the order $\Theta\left(n^{r-1}\right)$ are known if $r \geq 4$ (see [2, 3]). So Tuza and Voloshin [8] proposed the following problem:

Problem. Given a finite set $S$ of positive integers, determine or estimate the minimum numbers of $(\mathcal{C}$-, $\mathcal{D}$-, bi-) edges in a mixed (bi-) hypergraph whose feasible set is $S$.

In order to solve this problem for one-realizations of a given set, we need to deleting some $\mathcal{D}$-edges or $\mathcal{C}$-edges from the mixed hypergraphs constructed in [13]. Now we introduce the mixed hypergraphs.

In the rest we always assume that $S=\left\{n_{1}, n_{2}, \ldots, n_{s}\right\}$ is a set of integers with $2 \leq n_{s}<\cdots<n_{2}<n_{1}$ and $[n]$ is the set $\{1,2, \ldots, n\}$.

\section{Construction ([13]) Let}

$$
\begin{aligned}
X_{n_{1}, \ldots, n_{s}} & =\left\{\left(n_{1}, n_{2}, \ldots, n_{s}\right)\right\} \cup\left\{(\underbrace{i, i, \ldots, i}_{s}) \mid i \in\left[n_{s}-1\right]\right\} \\
& \cup \bigcup_{t=2}^{s} \bigcup_{j=n_{t}}^{n_{t-1}-1}\left\{(\underbrace{j, \ldots, j}_{t-1}, n_{t}, n_{t+1}, \ldots, n_{s}),(\underbrace{j, \ldots, j}_{t-1}, \underbrace{1, \ldots, 1}_{s-t+1})\right\}, \\
\mathcal{D}_{n_{1}, \ldots, n_{s}} & =\left\{\left\{\left(x_{1}, x_{2}, \ldots, x_{s}\right),\left(y_{1}, y_{2}, \ldots, y_{s}\right)\right\} \mid x_{i} \neq y_{i}, i \in[s]\right\}, \\
\mathcal{C}_{n_{1}, \ldots, n_{s}} & =\left\{\left\{\left(x_{1}, \ldots, x_{s}\right),\left(y_{1}, \ldots, y_{s}\right),\left(z_{1}, \ldots, z_{s}\right)\right\}||\left\{x_{j}, y_{j}, z_{j}\right\} \mid=2, j \in[s]\right\} .
\end{aligned}
$$

By [13, Theorems 1.1, 2.4 and 2.5] the hypergraph $\mathcal{H}_{n_{1}, \ldots, n_{s}}=\left(X_{n_{1}, \ldots, n_{s}}, \mathcal{C}_{n_{1}, \ldots, n_{s}}, \mathcal{D}_{n_{1}, \ldots, n_{s}}\right)$ (resp. $\left.\mathcal{G}_{n_{1}, \ldots, n_{s}}=\mathcal{H}_{n_{1}, \ldots, n_{s}}\left[X_{n_{1}, \ldots, n_{s}} \backslash\left\{\left(n_{2}, 1, \ldots, 1\right)\right\}\right]\right)$ is a smallest one-realization of $S$ when $n_{1}-1 \notin S$ (resp. $n_{1}-1 \in S$ ).

By finding some spanning sub-hypergraphs of $\mathcal{H}_{n_{1}, \ldots, n_{s}}$ and $\mathcal{G}_{n_{1}, \ldots, n_{s}}$, we determine the minimum number of $\mathcal{D}$-deges (resp. $\mathcal{C}$-edges) of one-realizations of $S$ as follows.

Theorem 1.1 Let $\delta_{\mathcal{D}}(S)$ denote the minimum number of $\mathcal{D}$-edges of one-realizations of $S$. Then

$$
\delta_{\mathcal{D}}(S)= \begin{cases}\frac{n_{1}\left(n_{1}-1\right)}{2}, & \text { if } n_{1}-1 \notin S, \\ \frac{n_{1}\left(n_{1}-1\right)}{2}-1, & \text { if } n_{1}-1 \in S .\end{cases}
$$

Theorem 1.2 Let $\delta_{\mathcal{C}}(S)$ be the minimum number of $\mathcal{C}$-edges of one-realizations of $S$. Then

$$
\delta_{\mathcal{C}}(S)= \begin{cases}2 n_{1}-2 n_{s}, & \text { if } n_{1}-1, n_{s}+1 \notin S, \\ 2 n_{1}-2 n_{s}-2, & \text { if } n_{1}-1, n_{s}+1 \in S, \\ 2 n_{1}-2 n_{s}-1, & \text { otherwise. }\end{cases}
$$




\section{Proof of Theorem 1.1}

We first show that the number $\delta_{\mathcal{D}}(S)$ given in Theorem 1.1 is a lower bound on the minimum number of $\mathcal{D}$-edges of one-realizations of $S$.

\section{Lemma 2.1}

$$
\delta_{\mathcal{D}}(S) \geq \begin{cases}\frac{n_{1}\left(n_{1}-1\right)}{2}, & \text { if } n_{1}-1 \notin S, \\ \frac{n_{1}\left(n_{1}-1\right)}{2}-1, & \text { if } n_{1}-1 \in S .\end{cases}
$$

Proof. Let $\mathcal{H}$ be a one-realization of $S$ and $c=\left\{C_{1}, C_{2}, \ldots, C_{n_{1}}\right\}$ be a strict $n_{1^{-}}$ coloring of $\mathcal{H}$.

Case 1. $n_{1}-1 \notin S$. If there exist two color classes $C_{i}$ and $C_{j}$ such that $\mathcal{H}\left[C_{i} \cup C_{j}\right]$ has no $\mathcal{D}$-edges, then we can color the vertices in $C_{i} \cup C_{j}$ with a common color and get a strict $\left(n_{1}-1\right)$-coloring of $\mathcal{H}$, a contradiction. Hence, the first inequality holds.

Case 2. $n_{1}-1 \in S$. That is to say, $n_{2}=n_{1}-1$. If there exist two distinct pairs of color classes, say $C_{i}, C_{j}$ and $C_{k}, C_{l}$, such that both $\mathcal{H}\left[C_{i} \cup C_{j}\right]$ and $\mathcal{H}\left[C_{k} \cup C_{l}\right]$ have no $\mathcal{D}$-edges, then we can color the vertices in $C_{i} \cup C_{j}$ with a common color and get a strict $n_{2}$-coloring of $\mathcal{H}$; and color the vertices in $C_{k} \cup C_{l}$ with a common color and get another strict $n_{2}$-coloring of $\mathcal{H}$, a contradiction. Hence, the second inequality holds.

Next we construct mixed hypergraphs which meet the bounds in Lemma 2.1, Let

$$
\begin{aligned}
\mathcal{D}_{n_{1}, \ldots, n_{s}}^{*} & =\left\{\{(i, i, \ldots, i),(j, j, \ldots, j)\} \mid i \neq j, i, j \in\left[n_{s}-1\right]\right\} \\
\cup & \left\{\left\{(i, \ldots, i),\left(x_{1}, \ldots, x_{s-1}, n_{s}\right)\right\} \mid i \in\left[n_{s}-1\right]\right\} \\
\cup & \left\{\left\{\left(x_{1}, \ldots, x_{s-1}, 1\right),\left(y_{1}, \ldots, y_{s-1}, n_{s}\right)\right\} \mid x_{i}<y_{i}, i \in[s-1]\right\} .
\end{aligned}
$$

Then $\mathcal{H}_{n_{1}, \ldots, n_{s}}^{*}=\left(X_{n_{1}, \ldots, n_{s}}, \mathcal{C}_{n_{1}, \ldots, n_{s}}, \mathcal{D}_{n_{1}, \ldots, n_{s}}^{*}\right)$ is a spanning sub-hypergraph of $\mathcal{H}_{n_{1}, \ldots, n_{s}}$ with $\frac{n_{1}\left(n_{1}-1\right)}{2} \mathcal{D}$-edges.

By [13, Theorem 2.4] all the strict colorings of $\mathcal{H}_{n_{1}, \ldots, n_{s}}$ are as follows:

$$
c_{i}^{s}=\left\{X_{i 1}^{s}, X_{i 2}^{s}, \ldots, X_{i n_{i}}^{s}\right\}, \quad i \in[s],
$$

where $X_{i j}^{s}=\left\{\left(x_{1}, x_{2}, \ldots, x_{s}\right) \in X_{n_{1}, \ldots, n_{s}} \mid x_{i}=j\right\}, j=1,2, \ldots, n_{i}$. Therefore, $c_{1}^{s}, \ldots, c_{s}^{s}$ are strict colorings of $\mathcal{H}_{n_{1}, \ldots, n_{s}}^{*}$.

In the following we shall prove by induction on $s$ that $c_{1}^{s}, \ldots, c_{s}^{s}$ are all the strict colorings of $\mathcal{H}_{n_{1}, \ldots, n_{s}}^{*}$, which follows that $\mathcal{H}_{n_{1}, \ldots, n_{s}}^{*}$ is a one-realization of $S$.

Lemma 2.2 $\mathcal{H}_{n_{1}, n_{2}}^{*}$ is a one-realization of $\left\{n_{1}, n_{2}\right\}$.

Proof. Let $c=\left\{C_{1}, C_{2}, \ldots, C_{m}\right\}$ be a strict coloring of $\mathcal{H}_{n_{1}, n_{2}}^{*}$. The vertices $(1,1),(2,2), \ldots,\left(n_{2}, n_{2}\right)$ fall into distinct color classes, say $(i, i) \in C_{i}, i \in\left[n_{2}\right]$. From the $\mathcal{C}$-edge $\left\{\left(n_{2}, 1\right),(1,1),\left(n_{2}, n_{2}\right)\right\}$, we have $\left(n_{2}, 1\right) \in C_{1} \cup C_{n_{2}}$.

Case 1. $\left(n_{2}, 1\right) \in C_{1}$. For any $k \in\left[n_{1}-n_{2}\right]$, from the $\mathcal{D}$-edge $\left\{\left(n_{2}, 1\right),\left(n_{2}+k, n_{2}\right)\right\}$ and the $\mathcal{C}$-edge $\left\{\left(n_{2}+k, n_{2}\right),\left(n_{2}, 1\right),\left(n_{2}, n_{2}\right)\right\}$, we have $\left(n_{2}+k, n_{2}\right) \in C_{n_{2}}$. For any $k \in\left[n_{1}-n_{2}-1\right]$, the $\mathcal{C}$-edge $\left\{\left(n_{2}+k, 1\right),\left(n_{2}, 1\right),\left(n_{2}+k, n_{2}\right)\right\}$ and the $\mathcal{D}$-edge $\left\{\left(n_{2}+\right.\right.$ $\left.k, 1),\left(n_{1}, n_{2}\right)\right\}$ imply that $\left(n_{2}+k, 1\right) \in C_{1}$. Therefore, $c=c_{2}^{2}$.

Case 2. $\left(n_{2}, 1\right) \in C_{n_{2}}$. The $\mathcal{D}$-edges $\left\{\left(n_{2}, 1\right),\left(n_{2}+1, n_{2}\right)\right\},\left\{(i, i),\left(n_{2}+1, n_{2}\right)\right\}, i \in$ $\left[n_{2}-1\right]$ imply that $\left(n_{2}+1, n_{2}\right) \notin C_{i}$ for any $i \in\left[n_{2}\right]$. Suppose $\left(n_{2}+1, n_{2}\right) \in C_{n_{2}+1}$. Since $\left\{\left(n_{2}+1,1\right),\left(n_{2}, 1\right),\left(n_{2}+1, n_{2}\right)\right\},\left\{\left(n_{2}+1,1\right),\left(n_{2}+1, n_{2}\right),(1,1)\right\}$ are $\mathcal{C}$-edges, $\left(n_{2}+1,1\right) \in C_{n_{2}+1}$. Similarly, $\left(n_{2}+k, n_{2}\right),\left(n_{2}+k, 1\right) \in C_{n_{2}+k}$ for any $k \in\left[n_{1}-n_{2}-1\right]$ and $\left(n_{1}, n_{2}\right) \in C_{n_{1}}$. Therefore, $c=c_{1}^{2}$. 
Theorem 2.3 $\mathcal{H}_{n_{1}, \ldots, n_{s}}^{*}$ is a one-realization of $S$.

Proof. By Lemma 2.2 the conclusion is true for $s=2$. Suppose that $s \geq 3$, and the conclusion is true for the case of $s-1$. Let $X^{\prime}=\left\{\left(x_{1}, x_{2}, \ldots, x_{s}\right) \in X_{n_{1}, \ldots, n_{s}} \mid x_{1}=x_{2}\right\}$. Then $\mathcal{H}^{*}=\mathcal{H}_{n_{1}, \ldots, n_{s}}^{*}\left[X^{\prime}\right]$ is isomorphic to $\mathcal{H}_{n_{2}, \ldots, n_{s}}^{*}$. By induction, all the strict colorings of $\mathcal{H}^{*}$ are as follows:

$$
c_{i}^{\prime}=\left\{X_{i 1}^{\prime}, X_{i 2}^{\prime}, \ldots, X_{i n_{i}}^{\prime}\right\}, \quad i \in[s] \backslash\{1\},
$$

where $X_{i j}^{\prime}=X^{\prime} \cap X_{i j}^{s}, j \in\left[n_{i}\right]$.

For any strict coloring $c=\left\{C_{1}, C_{2}, \ldots, C_{m}\right\}$ of $\mathcal{H}_{n_{1}, \ldots, n_{s}}^{*}$, the vertices $(1,1, \ldots, 1)$, $(2,2, \ldots, 2), \ldots,\left(n_{s}, n_{s}, \ldots, n_{s}\right)$ fall into distinct color classes, say $(i, i, \ldots, i) \in C_{i}, i \in$ $\left[n_{s}\right]$. There are the following two possible cases.

Case 1. $\left.c\right|_{X^{\prime}}=c_{2}^{\prime}$. That is to say, for any $i \in\left[n_{2}\right]$ and any $\left(i, i, x_{3}, \ldots, x_{s}\right) \in$ $X^{\prime},\left(i, i, x_{3}, \ldots, x_{s}\right) \in C_{i}$. The $\mathcal{C}$-edge $\left\{(1,1, \ldots, 1),\left(n_{2}, 1, \ldots, 1\right),\left(n_{2}, n_{2}, n_{3}, \ldots, n_{s}\right)\right\}$ implies that $\left(n_{2}, 1, \ldots, 1\right) \in C_{1} \cup C_{n_{2}}$.

Case 1.1. $\left(n_{2}, 1, \ldots, 1\right) \in C_{1}$. For any $k \in\left[n_{1}-n_{2}\right]$, from the $\mathcal{C}$-edge $\left\{\left(n_{2}+\right.\right.$ $\left.\left.k, n_{2}, n_{3}, \ldots, n_{s}\right),\left(n_{2}, 1, \ldots, 1\right),\left(n_{2}, n_{2}, n_{3}, \ldots, n_{s}\right)\right\}$ and the $\mathcal{D}$-edge $\left\{(1,1, \ldots, 1),\left(n_{2}+\right.\right.$ $\left.\left.k, n_{2}, n_{3}, \ldots, n_{s}\right)\right\}$, we observe $\left(n_{2}+k, n_{2}, n_{3}, \ldots, n_{s}\right) \in C_{n_{2}}$. For any $k \in\left[n_{1}-n_{2}-\right.$ $1]$, by the $\mathcal{C}$-edge $\left\{\left(n_{2}+k, 1, \ldots, 1\right),\left(n_{2}, n_{2}, n_{3}, \ldots, n_{s}\right),\left(n_{2}, 1, \ldots, 1\right)\right\}$ and the $\mathcal{D}$-edge $\left\{\left(n_{2}+k, 1, \ldots, 1\right),\left(n_{1}, n_{2}, n_{3}, \ldots, n_{s}\right)\right\}$, we obtain $\left(n_{2}+k, 1, \ldots, 1\right) \in C_{1}$. Therefore, $c=c_{2}^{s}$.

Case 1.2. $\left(n_{2}, 1, \ldots, 1\right) \in C_{n_{2}}$. The fact that $\left\{(i, i, \ldots, i),\left(n_{2}+1, n_{2}, \ldots, n_{s}\right)\right\}$ is a $\mathcal{D}$ edge follows that $\left(n_{2}+1, n_{2}, \ldots, n_{s}\right) \notin C_{i}$ for any $i \in\left[n_{s}-1\right]$. For each $t \in[s] \backslash\{1,2\}$ and $k \in\left[n_{t-1}-n_{t}-1\right] \cup\{0\}$, the $\mathcal{D}$-edge $\left\{(\underbrace{n_{t}+k, \ldots, n_{t}+k}_{t-1}, 1, \ldots, 1),\left(n_{2}+1, n_{2}, \ldots, n_{s}\right)\right\}$ implies that $\left(n_{2}+1, n_{2}, \ldots, n_{s}\right) \notin C_{n_{t}+k}$. Since $\left\{\left(n_{2}, 1, \ldots, 1\right),\left(n_{2}+1, n_{2}, \ldots, n_{s}\right)\right\}$ is a $\mathcal{D}$-edge, $\left(n_{2}+1, n_{2}, \ldots, n_{s}\right) \notin C_{n_{2}}$. Suppose $\left(n_{2}+1, n_{2}, \ldots, n_{s}\right) \in C_{n_{2}+1}$. From the $\mathcal{C}$ edges $\left\{\left(n_{2}+1,1, \ldots, 1\right),\left(n_{2}+1, n_{2}, \ldots, n_{s}\right),\left(n_{2}, 1, \ldots, 1\right)\right\}$ and $\left\{\left(n_{2}+1,1, \ldots, 1\right),\left(n_{2}+\right.\right.$ $\left.\left.1, n_{2}, \ldots, n_{s}\right),(1,1, \ldots, 1)\right\}$, we have $\left(n_{2}+1,1, \ldots, 1\right) \in C_{n_{2}+1}$. Similarly, for any $k \in$ $\left[n_{1}-n_{2}-1\right],\left(n_{2}+k, 1, \ldots, 1\right),\left(n_{2}+k, n_{2}, \ldots, n_{s}\right) \in C_{n_{2}+k}$ and $\left(n_{1}, n_{2}, \ldots, n_{s}\right) \in C_{n_{1}}$. Hence, $c=c_{1}^{s}$.

Case 2. $\left.c\right|_{X^{\prime}}=c_{t}^{\prime}$ for some $t \in[s] \backslash\{1,2\}$. In this case, we observe $\left(n_{2}-1, n_{2}-\right.$ $\left.1, n_{3}, \ldots, n_{s}\right) \in C_{n_{t}}$ and $\left(n_{2}-1, n_{2}-1,1, \ldots, 1\right) \in C_{1}$. For any $k \in\left[n_{1}-n_{2}\right]$, from the $\mathcal{C}$-edge $\left\{\left(n_{2}+k, n_{2}, \ldots, n_{s}\right),\left(n_{2}-1, n_{2}-1, n_{3}, \ldots, n_{s}\right),\left(n_{2}-1, n_{2}-1,1, \ldots, 1\right)\right\}$ and the $\mathcal{D}$-edge $\left\{(1,1, \ldots, 1),\left(n_{2}+k, n_{2}, \ldots, n_{s}\right)\right\}$, we have $\left(n_{2}+k, n_{2}, \ldots, n_{s}\right) \in C_{n_{t}}$. Furthermore, the $\mathcal{C}$-edge $\left\{\left(n_{2}+k, 1, \ldots, 1\right),\left(n_{2}+k, n_{2}, \ldots, n_{s}\right),(1,1, \ldots, 1)\right\}$ and the $\mathcal{D}$-edge $\left\{\left(n_{2}+k, 1, \ldots, 1\right),\left(n_{1}, n_{2}, \ldots, n_{s}\right)\right\}$ imply that $\left(n_{2}+k, 1, \ldots, 1\right) \in C_{1}$ for any $k \in\left[n_{1}-n_{2}-1\right] \cup\{0\}$. Therefore, $c=c_{t}^{s}$.

When $n_{1}-1 \in S$, that is to say, $n_{2}=n_{1}-1$, let $X^{\prime \prime}=X_{n_{1}, \ldots, n_{s}} \backslash\left\{\left(n_{2}, 1, \ldots, 1\right)\right\}$. Then $\mathcal{G}_{n_{1}, \ldots, n_{s}}^{*}=\mathcal{H}_{n_{1}, \ldots, n_{s}}^{*}\left[X^{\prime \prime}\right]$ is a spanning sub-hypergraph of $\mathcal{G}_{n_{1}, \ldots, n_{s}}$ with $\frac{n_{1}\left(n_{1}-1\right)}{2}-1$ $\mathcal{D}$-edges. Note that for any $i \in[s]$,

$$
c_{i}^{\prime \prime}=\left\{X_{i 1}^{\prime \prime}, X_{i 2}^{\prime \prime}, \ldots, X_{i n_{i}}^{\prime \prime}\right\}
$$

is a strict $n_{i}$-coloring of $\mathcal{G}_{n_{1}, \ldots, n_{s}}^{*}$, where $X_{i j}^{\prime \prime}=X^{\prime \prime} \cap X_{i j}^{s}, j=1,2, \ldots, n_{i}$.

Theorem 2.4 If $n_{1}-1 \in S$, then $\mathcal{G}_{n_{1}, \ldots, n_{s}}^{*}$ is a one-realization of $S$. 
Proof. Referring to the proof of Theorem 2.3, all the strict colorings of $\mathcal{H}^{*}$ are $c_{2}^{\prime}, c_{3}^{\prime}, \ldots, c_{s}^{\prime}$. For any strict coloring $c=\left\{C_{1}, C_{2}, \ldots, C_{m}\right\}$ of $\mathcal{G}_{n_{1}, \ldots, n_{s}}^{*}$, we focus on the restriction of $c$ on $X^{\prime}$ and get the following two possible cases.

Case 1. $\left.c\right|_{X^{\prime}}=c_{2}^{\prime}$. That is to say, $\left(i, i, x_{3}, \ldots, x_{s}\right) \in C_{i}$ for any $i \in\left[n_{2}\right]$ and $\left(i, i, x_{3}, \ldots, x_{s}\right) \in X^{\prime}$. By the proof of Theorem 2.3 we have $\left(n_{1}, n_{2}, n_{3}, \ldots, n_{s}\right) \notin C_{j}$ for any $j \in\left[n_{2}-1\right]$. If $\left(n_{1}, n_{2}, n_{3}, \ldots, n_{s}\right) \in C_{n_{2}}$, then $c=c_{2}^{\prime \prime}$; If $\left(n_{1}, n_{2}, n_{3}, \ldots, n_{s}\right) \notin C_{n_{2}}$, then $\left(n_{1}, n_{2}, n_{3}, \ldots, n_{s}\right) \in C_{n_{1}}$, which implies that $c=c_{1}^{\prime \prime}$.

Case 2. $\left.c\right|_{X^{\prime}}=c_{t}^{\prime}$ for some $t \in[s] \backslash\{1,2\}$. That is to say, $\left(n_{t}, \ldots, n_{t}, n_{t+1}, \ldots, n_{s}\right) \in$ $C_{n_{t}}$ and $(\underbrace{n_{t}, \ldots, n_{t}}_{t-1}, 1, \ldots, 1) \in C_{1}$. The $\mathcal{D}$-edge $\left\{(1,1, \ldots, 1),\left(n_{1}, n_{2}, \ldots, n_{s}\right)\right\}$ and the $\mathcal{C}$-edge $\left\{\left(n_{1}, n_{2}, \ldots, n_{s}\right),\left(n_{t}, \ldots, n_{t}, n_{t+1}, \ldots, n_{s}\right),\left(n_{t}, \ldots, n_{t}, 1, \ldots, 1\right)\right\}$ imply that $\left(n_{1}, n_{2}, \ldots, n_{s}\right) \in C_{n_{t}}$. Hence, $c=c_{t}^{\prime \prime}$.

By above discussion, $c_{1}^{\prime \prime}, c_{2}^{\prime \prime}, \ldots, c_{s}^{\prime \prime}$ are all the strict colorings of $\mathcal{G}_{n_{1}, \ldots, n_{s}}^{*}$. Therefore, $\mathcal{G}_{n_{1}, \ldots, n_{s}}^{*}$ is a one-realization of $S$.

Combining Lemma 2.1, Theorems 2.3 and 2.4, we complete the proof of Theorem 1.1

\section{Proof of Theorem 1.2}

In [13, we determined the minimum number of vertices of one-realizations of $S$.

Theorem 3.1 ([13]) Let $\delta(S)$ denote the minimum number of vertices of one-realizations of $S$. Then

$$
\delta(S)= \begin{cases}2 n_{1}-n_{s}, & \text { if } n_{1}-1 \notin S \\ 2 n_{1}-n_{s}-1, & \text { if } n_{1}-1 \in S\end{cases}
$$

Let $\mathcal{H}$ be a mixed hypergraph with a strict $m$-coloring $c=\left\{C_{1}, C_{2}, \ldots, C_{m}\right\}$. For any $i$, the pair graph $G_{i}$ of $C_{i}$ under $c$ has $C_{i}$ as its vertex set, and two vertices $x$ and $y$ are adjacent if there exists a $\mathcal{C}$-edge $C$ such that $C \cap C_{i}=\{x, y\}$ and $\left|C \cap C_{j}\right| \leq 1$ for any $j \in[m] \backslash\{i\}$. Note that the definition of pair graphs is different from that in [2].

The following result shows that the number $\delta_{\mathcal{C}}(S)$ given in Theorem 1.2 is a lower bound on the minimum number of $\mathcal{C}$-edges of one-realizations of $S$.

\section{Lemma 3.2}

$$
\delta_{\mathcal{C}}(S) \geq \begin{cases}\delta(S)-n_{s}, & \text { if } n_{s}+1 \notin S \\ \delta(S)-n_{s}-1, & \text { if } n_{s}+1 \in S\end{cases}
$$

Proof. Let $\mathcal{H}=(X, \mathcal{C}, \mathcal{D})$ be a one-realization of $S$ and $c=\left\{C_{1}, C_{2}, \ldots, C_{n_{s}}\right\}$ be a strict $n_{s}$-coloring of $\mathcal{H}$. For any $i \in\left[n_{s}\right]$, let $G_{i}=\left(C_{i}, E_{i}\right)$ be the pair graph of $C_{i}$ under $c$.

Case 1. $n_{s}+1 \notin S$. If $G_{i}$ is a disconnected graph with $G_{i}^{1}=\left(C_{i}^{1}, E_{i}^{1}\right)$ as one of its connected components, then $\left\{C_{1}, \ldots, C_{i-1}, C_{i}^{1},\left(C_{i} \backslash C_{i}^{1}\right), C_{i+1}, \ldots, C_{n_{s}}\right\}$ is a strict $\left(n_{s}+1\right)$-coloring of $\mathcal{H}$, a contradiction. Therefore, each $G_{i}$ is connected; and so $\left|E_{i}\right| \geq$ $\left|C_{i}\right|-1$. Since every $\mathcal{C}$-edge of $\mathcal{H}$ corresponds to at most one edge in the $n_{s}$ pair graphs, we have $\delta_{\mathcal{C}}(S) \geq\left(\left|C_{1}\right|-1\right)+\left(\left|C_{2}\right|-1\right)+\cdots+\left(\left|C_{n_{s}}\right|-1\right)=|X|-n_{s} \geq \delta(S)-n_{s}$.

Case 2. $n_{s}+1 \in S$. Assume that there exist two disconnected pair graphs, say $G_{1}$ and $G_{2}$. Pick a connected component $G_{1}^{1}=\left(C_{1}^{1}, E_{1}^{1}\right)$ of $G_{1}$, and a connected 
component $G_{2}^{1}=\left(C_{2}^{1}, E_{2}^{1}\right)$ of $G_{2}$. Then $\left\{C_{1}^{1},\left(C_{1} \backslash C_{1}^{1}\right), C_{2}, \ldots, C_{n_{s}}\right\}$ and $\left\{C_{1}, C_{2}^{1},\left(C_{2} \backslash\right.\right.$ $\left.\left.C_{2}^{1}\right), C_{3}, \ldots, C_{n_{s}}\right\}$ are two distinct strict $\left(n_{s}+1\right)$-colorings of $\mathcal{H}$, a contradiction. If there exists a pair graph, say $G_{1}$, with at least three connected components $G_{1}^{1}=\left(C_{1}^{1}, E_{1}^{1}\right)$, $G_{1}^{2}=\left(C_{1}^{2}, E_{1}^{2}\right)$ and $G_{1}^{3}=\left(C_{1}^{3}, E_{1}^{3}\right)$, then $\left\{C_{1}^{1},\left(C_{1} \backslash C_{1}^{1}\right), C_{2}, \ldots, C_{n_{s}}\right\},\left\{C_{1}^{2},\left(C_{1} \backslash\right.\right.$ $\left.\left.C_{1}^{2}\right), C_{2}, \ldots, C_{n_{s}}\right\}$ are two distinct strict $\left(n_{s}+1\right)$-colorings of $\mathcal{H}$, a contradiction. Hence, there exists at most one pair graph with two connected components. It follows that $\mathcal{H}$ has at least $\delta(S)-n_{s}-1 \mathcal{C}$-edges.

Next we construct mixed hypergraphs which meet the bounds in Lemma 3.2. Let

$$
\begin{aligned}
& \mathcal{C}_{n_{1}, \ldots, n_{s}}^{\star} n_{t-1-1} \\
& =\bigcup_{t=2} \bigcup_{j=n_{t}+1}^{s}\left\{\left\{\left(j, \ldots, j, n_{t}, \ldots, n_{s}\right),(\underbrace{j, \ldots, j}_{t-1}, 1, \ldots, 1),(\underbrace{j-1, \ldots, j-1}_{t-1}, 1, \ldots, 1)\right\}\right\} \\
& \left.\cup \bigcup_{t=2}^{s} \bigcup_{j=n_{t}}^{n_{t-1}-1}\left\{(\underbrace{j, \ldots, j}_{t-1}, 1, \ldots, 1),\left(j, \ldots, j, n_{t}, \ldots, n_{s}\right),\left(j+1, \ldots, j+1, n_{t}, \ldots, n_{s}\right)\right\}\right\} \\
& \cup \bigcup_{t=2}^{s}\left\{\left\{\left(n_{t}, \ldots, n_{t}, n_{t}, \ldots, n_{s}\right),(\underbrace{n_{t}, \ldots, n_{t}}_{t-1}, 1, \ldots, 1),(1,1, \ldots, 1)\right\}\right\} .
\end{aligned}
$$

Then $\mathcal{H}_{n_{1}, \ldots, n_{s}}^{\star}=\left(X_{n_{1}, \ldots, n_{s}}, \mathcal{C}_{n_{1}, \ldots, n_{s}}^{\star}, \mathcal{D}_{n_{1}, \ldots, n_{s}}\right)$ is a spanning sub-hypergraph of $\mathcal{H}_{n_{1}, \ldots, n_{s}}$ with $2 n_{1}-2 n_{s} \mathcal{C}$-edges. Therefore, $c_{1}^{s}, c_{2}^{s}, \ldots, c_{s}^{s}$ in (11) are strict colorings of $\mathcal{H}_{n_{1}, \ldots, n_{s}}^{\star}$.

In the following, we shall prove by induction on $s$ that $c_{1}^{s}, \ldots, c_{s}^{s}$ are all the strict colorings of $\mathcal{H}_{n_{1}, \ldots, n_{s}}^{\star}$, which follows that $\mathcal{H}_{n_{1}, \ldots, n_{s}}^{\star}$ is a one-realization of $S$.

Lemma 3.3 $\mathcal{H}_{n_{1}, n_{2}}^{\star}$ is a one-realization of $\left\{n_{1}, n_{2}\right\}$.

Proof. Suppose $c=\left\{C_{1}, C_{2}, \ldots, C_{m}\right\}$ is a strict coloring of $\mathcal{H}_{n_{1}, n_{2}}^{\star}$. The vertices $(1,1),(2,2), \ldots,\left(n_{2}, n_{2}\right)$ fall into distinct color classes, say $(i, i) \in C_{i}, i \in\left[n_{2}\right]$. From the $\mathcal{C}$-edge $\left\{\left(n_{2}, 1\right),\left(n_{2}, n_{2}\right),(1,1)\right\}$, we have $\left(n_{2}, 1\right) \in C_{1} \cup C_{n_{2}}$.

Case 1. $\left(n_{2}, 1\right) \in C_{1}$. The $\mathcal{C}$-edge $\left\{\left(n_{2}, 1\right),\left(n_{2}, n_{2}\right),\left(n_{2}+1, n_{2}\right)\right\}$ and the $\mathcal{D}$-edge $\left\{(1,1),\left(n_{2}+1, n_{2}\right)\right\}$ imply that $\left(n_{2}+1, n_{2}\right) \in C_{n_{2}}$. From the $\mathcal{D}$-edge $\left\{\left(n_{2}+1,1\right),\left(n_{2}, n_{2}\right)\right\}$ and the $\mathcal{C}$-edge $\left\{\left(n_{2}+1,1\right),\left(n_{2}+1, n_{2}\right),\left(n_{2}, 1\right)\right\}$, we have $\left(n_{2}+1,1\right) \in C_{1}$. Similarly, for any $k \in\left[n_{1}-n_{2}-1\right]$, we have $\left(n_{2}+k, 1\right) \in C_{1},\left(n_{2}+k, n_{2}\right) \in C_{n_{2}}$ and $\left(n_{1}, n_{2}\right) \in C_{n_{2}}$. Therefore, $c=c_{2}^{2}$.

Case 2. $\left(n_{2}, 1\right) \in C_{n_{2}}$. Observe $\left(n_{2}+1, n_{2}\right) \notin C_{i}$ for each $i \in\left[n_{2}-1\right]$. The $\mathcal{D}$-edge $\left\{\left(n_{2}, 1\right),\left(n_{2}+1, n_{2}\right)\right\}$ implies that $\left(n_{2}+1, n_{2}\right) \notin C_{n_{2}}$. Suppose $\left(n_{2}+1, n_{2}\right) \in C_{n_{2}+1}$. From the $\mathcal{C}$-edge $\left\{\left(n_{2}+1,1\right),\left(n_{2}+1, n_{2}\right),\left(n_{2}, 1\right)\right\}$ and the $\mathcal{D}$-edge $\left\{\left(n_{2}+1,1\right),\left(n_{2}, n_{2}\right)\right\}$, we get $\left(n_{2}+1,1\right) \in C_{n_{2}+1}$. Similarly, $\left(n_{2}+k, n_{2}\right),\left(n_{2}+k, 1\right) \in C_{n_{2}+k}$ for any $k \in\left[n_{1}-n_{2}-1\right]$ and $\left(n_{1}, n_{2}\right) \in C_{n_{1}}$. Therefore, $c=c_{1}^{2}$.

Theorem 3.4 $\mathcal{H}_{n_{1}, \ldots, n_{s}}^{\star}$ is a one-realization of $S$.

Proof. By Lemma 3.3 the conclusion is true for $s=2$. Suppose $s \geq 3$, and the conclusion is true for the case of $s-1$. Let $X^{\prime}=\left\{\left(x_{1}, x_{2}, \ldots, x_{s}\right) \in X_{n_{1}, \ldots, n_{s}} \mid x_{1}=x_{2}\right\}$. Then $\mathcal{H}^{\star}=\mathcal{H}_{n_{1}, \ldots, n_{s}}^{\star}\left[X^{\prime}\right]$ is isomorphic to $\mathcal{H}_{n_{2}, n_{3}, \ldots, n_{s}}^{\star}$. By induction, all the strict colorings of $\mathcal{H}^{\star}$ are $c_{2}^{\prime}, c_{3}^{\prime}, \ldots, c_{s}^{\prime}$ in (2).

For any strict coloring $c=\left\{C_{1}, C_{2}, \ldots, C_{m}\right\}$ of $\mathcal{H}_{n_{1}, \ldots, n_{s}}^{\star}$, the vertices $(1,1, \ldots, 1)$, $(2,2, \ldots, 2), \ldots,\left(n_{s}, n_{s}, \ldots, n_{s}\right)$ fall into distinct color classes, say $(i, i, \ldots, i) \in C_{i}, i \in$ $\left[n_{s}\right]$. We focus on the restriction of $c$ on $X^{\prime}$ and get the following two possible cases. 
Case 1. $\left.c\right|_{X^{\prime}}=c_{2}^{\prime}$. That is to say, for any $i \in\left[n_{2}\right]$ and any $\left(i, i, x_{3}, \ldots, x_{s}\right) \in$ $X^{\prime},\left(i, i, x_{3}, \ldots, x_{s}\right) \in C_{i}$. The $\mathcal{C}$-edge $\left\{\left(n_{2}, 1, \ldots, 1\right),\left(n_{2}, n_{2}, n_{3}, \ldots, n_{s}\right),(1,1, \ldots, 1)\right\}$ implies that $\left(n_{2}, 1, \ldots, 1\right) \in C_{1} \cup C_{n_{2}}$.

Case 1.1. $\left(n_{2}, 1, \ldots, 1\right) \in C_{1}$. From the $\mathcal{D}$-edge $\left\{(1,1, \ldots, 1),\left(n_{2}+1, n_{2}, n_{3}, \ldots, n_{s}\right)\right\}$ and the $\mathcal{C}$-edge $\left\{\left(n_{2}, 1, \ldots, 1\right),\left(n_{2}, n_{2}, n_{3}, \ldots, n_{s}\right),\left(n_{2}+1, n_{2}, n_{3}, \ldots, n_{s}\right)\right\}$, we observe $\left(n_{2}+1, n_{2}, n_{3}, \ldots, n_{s}\right) \in C_{n_{2}}$. Then the $\mathcal{D}$-edge $\left\{\left(n_{2}+1,1, \ldots, 1\right),\left(n_{2}, n_{2}, n_{3}, \ldots, n_{s}\right)\right\}$ and the $\mathcal{C}$-edge $\left\{\left(n_{2}+1,1, \ldots, 1\right),\left(n_{2}+1, n_{2}, n_{3}, \ldots, n_{s}\right),\left(n_{2}, 1, \ldots, 1\right)\right\}$ imply that $\left(n_{2}+\right.$ $1,1, \ldots, 1) \in C_{1}$. Similarly, for any $k \in\left[n_{1}-n_{2}-1\right],\left(n_{2}+k, n_{2}, \ldots, n_{s}\right) \in C_{n_{2}},\left(n_{2}+\right.$ $k, 1, \ldots, 1) \in C_{1}$ and $\left(n_{1}, n_{2}, n_{3}, \ldots, n_{s}\right) \in C_{n_{2}}$. Therefore, $c=c_{2}^{s}$.

Case 1.2. $\left(n_{2}, 1, \ldots, 1\right) \in C_{n_{2}}$. The fact that $\left\{\left(n_{2}+1, n_{2}, \ldots, n_{s}\right),(i, i, \ldots, i)\right\}$ is a $\mathcal{D}$ edge follows that $\left(n_{2}+1, n_{2}, \ldots, n_{s}\right) \notin C_{i}$ for any $i \in\left[n_{s}-1\right]$. For any $t \in[s] \backslash\{1,2\}$ and $k \in\left[n_{t-1}-n_{t}-1\right] \cup\{0\}$, the $\mathcal{D}$-edge $\left\{\left(n_{2}+1, n_{2}, \ldots, n_{s}\right),(\underbrace{n_{t}+k, \ldots, n_{t}+k}_{t-1}, 1, \ldots, 1)\right\}$ implies that $\left(n_{2}+1, n_{2}, \ldots, n_{s}\right) \notin C_{n_{t}+k}$. Since $\left\{\left(n_{2}+1, n_{2}, \ldots, n_{s}\right),\left(n_{2}, 1, \ldots, 1\right)\right\}$ is a D -edge, $\left(n_{2}+1, n_{2}, \ldots, n_{s}\right) \notin C_{n_{2}}$. Suppose $\left(n_{2}+1, n_{2}, \ldots, n_{s}\right) \in C_{n_{2}+1}$. From the $\mathcal{C}$-edge $\left\{\left(n_{2}+1,1, \ldots, 1\right),\left(n_{2}+1, n_{2}, \ldots, n_{s}\right),\left(n_{2}, 1, \ldots, 1\right)\right\}$ and the $\mathcal{D}$-edge $\left\{\left(n_{2}+\right.\right.$ $\left.1,1, \ldots, 1),\left(n_{2}, n_{2}, \ldots, n_{s}\right)\right\}$, we have $\left(n_{2}+1,1, \ldots, 1\right) \in C_{n_{2}+1}$. Similarly, $\left(n_{2}+\right.$ $k, 1, \ldots, 1),\left(n_{2}+k, n_{2}, \ldots, n_{s}\right) \in C_{n_{2}+k}$ for any $k \in\left[n_{1}-n_{2}-1\right]$ and $\left(n_{1}, n_{2}, \ldots, n_{s}\right) \in$ $C_{n_{1}}$. Therefore, $c=c_{1}^{s}$.

Case 2. $\left.c\right|_{X^{\prime}}=c_{t}^{\prime}$ for some $t \in[s] \backslash\{1,2\}$. Then $\left(n_{2}, n_{2}, n_{3}, \ldots, n_{s}\right),\left(n_{2}-1, n_{2}-\right.$ $\left.1, n_{3}, \ldots, n_{s}\right) \in C_{n_{t}},\left(n_{2}-1, n_{2}-1,1, \ldots, 1\right) \in C_{1}$. From the $\mathcal{C}$-edge $\left\{\left(n_{2}, 1, \ldots, 1\right)\right.$, $\left.\left(n_{2}, n_{2}, n_{3}, \ldots, n_{s}\right),(1,1, \ldots, 1)\right\}$ and the $\mathcal{D}$-edge $\left\{\left(n_{2}, 1, \ldots, 1\right),\left(n_{2}-1, n_{2}-1, n_{3}, \ldots, n_{s}\right)\right\}$, we have $\left(n_{2}, 1, \ldots, 1\right) \in C_{1}$. From the $\mathcal{C}$-edge $\left\{\left(n_{2}, 1, \ldots, 1\right),\left(n_{2}, n_{2}, n_{3}, \ldots, n_{s}\right),\left(n_{2}+\right.\right.$ $\left.\left.1, n_{2}, \ldots, n_{s}\right)\right\}$ and the $\mathcal{D}$-edge $\left\{\left(n_{2}+1, n_{2}, \ldots, n_{s}\right),(1,1, \ldots, 1)\right\}$, we obtain $\left(n_{2}+\right.$ $\left.1, n_{2}, \ldots, n_{s}\right) \in C_{n_{t}}$. Then the $\mathcal{C}$-edge $\left\{\left(n_{2}+1,1, \ldots, 1\right),\left(n_{2}+1, n_{2}, \ldots, n_{s}\right),\left(n_{2}, 1, \ldots, 1\right)\right\}$ and the $\mathcal{D}$-edge $\left\{\left(n_{2}, n_{2}, \ldots, n_{s}\right),\left(n_{2}+1,1, \ldots, 1\right)\right\}$ imply that $\left(n_{2}+1,1, \ldots, 1\right) \in C_{1}$. Similarly, for any $k \in\left[n_{1}-n_{2}-1\right],\left(n_{2}+k, n_{2}, \ldots, n_{s}\right) \in C_{n_{t}},\left(n_{2}+k, 1, \ldots, 1\right) \in C_{1}$, and $\left(n_{1}, n_{2}, \ldots, n_{s}\right) \in C_{n_{t}}$. Hence, $c=c_{t}^{s}$.

When $n_{1}-1 \in S$, that is to say, $n_{1}=n_{2}+1$, let $\mathcal{G}_{n_{1}, \ldots, n_{s}}^{\star}=\mathcal{H}_{n_{1}, \ldots, n_{s}}^{\star}\left[X^{\prime \prime}\right]+e_{1}$, where $e_{1}=\left\{\left(n_{2}-1, n_{2}-1,1, \ldots, 1\right),\left(n_{2}-1, n_{2}-1, n_{3}, \ldots, n_{s}\right),\left(n_{1}, n_{2}, \ldots, n_{s}\right)\right\}$ and $X^{\prime \prime}=X_{n_{1}, \ldots, n_{s}} \backslash\left\{\left(n_{2}, 1, \ldots, 1\right)\right\}$. Then, $\mathcal{G}_{n_{1}, \ldots, n_{s}}^{\star}$ is a spanning sub-hypergraph of $\mathcal{G}_{n_{1}, \ldots, n_{s}}$ with $2 n_{1}-2 n_{s}-1 \mathcal{C}$-edges. Therefore, $c_{1}^{\prime \prime}, c_{2}^{\prime \prime}, \ldots, c_{s}^{\prime \prime}$ in (3) are strict colorings of $\mathcal{G}_{n_{1}, \ldots, n_{s}}^{\star}$.

Theorem 3.5 If $n_{1}=n_{2}+1$, then $\mathcal{G}_{n_{1}, \ldots, n_{s}}^{\star}$ is a one-realization of $S$.

Proof. Let $X^{\prime}=\left\{\left(x_{1}, x_{2}, \ldots, x_{s}\right) \in X_{n_{1}, \ldots, n_{s}} \mid x_{1}=x_{2}\right\}$ and $\mathcal{G}^{\star}=\mathcal{G}_{n_{1}, \ldots, n_{s}}^{\star}\left[X^{\prime}\right]$. Referring to the proof of Theorem 3.4, all the strict colorings of $\mathcal{H}^{\star}$ are $c_{2}^{\prime}, c_{3}^{\prime}, \ldots, c_{s}^{\prime}$ in (22). For any strict coloring $c=\left\{C_{1}, C_{2}, \ldots, C_{m}\right\}$ of $\mathcal{G}_{n_{1}, \ldots, n_{s}}^{\star}$, we focus on the restriction of $c$ on $X^{\prime}$ and get the following two possible cases.

Case 1. $\left.c\right|_{X^{\prime}}=c_{2}^{\prime}$. That is to say, $\left(i, i, x_{3}, \ldots, x_{s}\right) \in C_{i}$ for any $i \in\left[n_{2}\right]$ and $\left(i, i, x_{3}, \ldots, x_{s}\right) \in X^{\prime}$. By the same discussion as we do in Theorem 3.4 we have $\left(n_{1}, n_{2}, n_{3}, \ldots, n_{s}\right) \notin C_{j}$ for any $j \in\left[n_{2}-1\right]$. If $\left(n_{1}, n_{2}, n_{3}, \ldots, n_{s}\right) \in C_{n_{2}}$, then $c=c_{2}^{\prime \prime}$; If $\left(n_{1}, n_{2}, n_{3}, \ldots, n_{s}\right) \notin C_{n_{2}}$, then $\left(n_{1}, n_{2}, n_{3}, \ldots, n_{s}\right) \in C_{n_{1}}$ which follows that $c=c_{1}^{\prime \prime}$.

Case 2. $\left.c\right|_{X^{\prime}}=c_{t}^{\prime}$ for some $t \in[s] \backslash\{1,2\}$. Then $\left(n_{2}-1, n_{2}-1, n_{3}, \ldots, n_{s}\right) \in$ $C_{n_{t}}$ and $(1,1, \ldots, 1),\left(n_{2}-1, n_{2}-1,1, \ldots, 1\right) \in C_{1}$. From the $\mathcal{C}$-edge $\left\{\left(n_{2}-1, n_{2}-\right.\right.$ $\left.1,1, \ldots, 1),\left(n_{2}-1, n_{2}-1, n_{3}, \ldots, n_{s}\right),\left(n_{1}, n_{2}, \ldots, n_{s}\right)\right\}$ and the $\mathcal{D}$-edge $\left\{\left(n_{1}, n_{2}, \ldots, n_{s}\right)\right.$, $(1,1, \ldots, 1)\}$, we observe $\left(n_{1}, n_{2}, \ldots, n_{s}\right) \in C_{n_{t}}$. Hence, $c=c_{t}^{\prime \prime}$. 
When $n_{1}-1 \notin S$ and $n_{s}+1 \in S$, let $\mathcal{H}_{n_{1}, \ldots, n_{s}}^{\star 1}=\mathcal{H}_{n_{1}, \ldots, n_{s-2}, n_{s}}^{\star}-e_{2}$, where $e_{2}=\left\{\left(n_{s}, \ldots, n_{s}, 1\right),\left(n_{s}, \ldots, n_{s}, n_{s}\right),(1, \ldots, 1,1)\right\}$. Then $\mathcal{H}_{n_{1}, \ldots, n_{s}}^{\star 1}$ is a spanning subhypergraph of $\mathcal{H}_{n_{1}, \ldots, n_{s-2}, n_{s}}^{\star}$ with $2 n_{1}-2 n_{s}-1 \mathcal{C}$-edges. Note that, for any $i \in[s] \backslash\{s-1\}$,

$$
c_{i}^{s-1}=\left\{X_{i 1}^{s-1}, X_{i 2}^{s-1}, \ldots, X_{i n_{i}}^{s-1}\right\}
$$

is a strict $n_{i}$-coloring of $\mathcal{H}_{n_{1}, \ldots, n_{s}}^{\star 1}$, where $X_{i j}^{s-1}=\left\{\left(x_{1}, \ldots, x_{s-2}, x_{s}\right) \in X_{n_{1}, \ldots, n_{s-2}, n_{s}} \mid x_{i}=\right.$ $j\}, j=1,2, \ldots, n_{i}$.

Theorem 3.6 If $n_{1}-1 \notin S$ and $n_{s}+1 \in S$, then $\mathcal{H}_{n_{1}, \ldots, n_{s}}^{\star 1}$ is a one-realization of $S$.

Proof. Let $c=\left\{C_{1}, C_{2}, \ldots, C_{m}\right\}$ be a strict coloring of $\mathcal{H}_{n_{1}, \ldots, n_{s}}^{\star 1}$. Then the vertices $(i, i, \ldots, i), i=1,2, \ldots, n_{s}$ fall into distinct color classes. Suppose $(i, i, \ldots, i) \in C_{i}$ for any $i \in\left[n_{s}\right]$. Note that $\left(n_{s}, \ldots, n_{s}, 1\right) \notin C_{i}, i \in\left[n_{s}\right] \backslash\left\{1, n_{s}\right\}$, we get the following two possible cases.

Case 1. $\left(n_{s}, \ldots, n_{s}, 1\right) \in C_{1} \cup C_{n_{s}}$. By the same discussion in Theorem 3.4, we have $c \in\left\{c_{1}^{s-1}, \ldots, c_{s-2}^{s-1}, c_{s}^{s-1}\right\}$.

Case 2. $\left(n_{s}, \ldots, n_{s}, 1\right) \notin C_{i}, i \in\left[n_{s}\right]$. Suppose $\left(n_{s}, \ldots, n_{s}, 1\right) \in C_{n_{s-1}}$. Then the $\mathcal{D}$-edge $\left\{\left(n_{s}, \ldots, n_{s}, 1\right),\left(n_{s}+1, \ldots, n_{s}+1, n_{s}\right)\right\}$ and the $\mathcal{C}$-edge $\left\{\left(n_{s}+1, \ldots, n_{s}+\right.\right.$ $\left.\left.1, n_{s}\right),\left(n_{s}, \ldots, n_{s}, 1\right),\left(n_{s}, \ldots, n_{s}, n_{s}\right)\right\}$ imply that $\left(n_{s}+1, \ldots, n_{s}+1, n_{s}\right) \in C_{n_{s}}$. From the $\mathcal{C}$-edge $\left\{\left(n_{s}+1, \ldots, n_{s}+1,1\right),\left(n_{s}+1, \ldots, n_{s}+1, n_{s}\right),\left(n_{s}, \ldots, n_{s}, 1\right)\right\}$ and the $\mathcal{D}$-edge $\left\{\left(n_{s}, \ldots, n_{s}, n_{s}\right),\left(n_{s}+1, \ldots, n_{s}+1,1\right)\right\}$, we have $\left(n_{s}+1, \ldots, n_{s}+1,1\right) \in C_{n_{s-1}}$. Similarly, for any $k \in\left[n_{s-2}-n_{s}-1\right],\left(n_{s}+k, \ldots, n_{s}+k, n_{s}\right) \in C_{n_{s}},\left(n_{s}+k, \ldots, n_{s}+k, 1\right) \in$ $C_{n_{s-1}}$ and $\left(n_{s-2}, \ldots, n_{s-2}, n_{s}\right) \in C_{n_{s}}$. Since $\left\{\left(n_{s-2}, \ldots, n_{s-2}, 1,1\right),\left(n_{s}, \ldots, n_{s}, n_{s}\right)\right\}$ is a $\mathcal{D}$-edge and $\left\{\left(n_{s-2}, \ldots, n_{s-2}, 1,1\right),\left(n_{s-2}, \ldots, n_{s-2}, n_{s}\right),(1, \ldots, 1)\right\}$ is a $\mathcal{C}$-edge, we get $\left(n_{s-2}, \ldots, n_{s-2}, 1,1\right) \in C_{1}$. Similar to the discussion in Theorem 3.4, we have $\left(x_{1}, \ldots, x_{s-3}, 1,1\right) \in C_{1}$ and $\left(x_{1}, \ldots, x_{s-3}, n_{s-2}, n_{s}\right) \in C_{n_{s}}$ for any $\left(x_{1}, \ldots, x_{s-3}, 1,1\right)$, $\left(x_{1}, \ldots, x_{s-3}, n_{s-2}, n_{s}\right) \in X_{n_{1}, \ldots, n_{s-2}, n_{s}}$. Therefore, $c$ is a strict $n_{s-1}$-coloring of $\mathcal{H}_{n_{1}, \ldots, n_{s}}^{\star 1}$.

By the above discussion, we have that $\mathcal{H}_{n_{1}, \ldots, n_{s}}^{\star 1}$ is a one-realization of $S$.

When $n_{1}-1, n_{s}+1 \in S$, let $X^{\prime \prime \prime}=X_{n_{1}, \ldots, n_{s-2}, n_{s}} \backslash\left\{\left(n_{2}, 1, \ldots, 1\right)\right\}$ and $\mathcal{H}_{n_{1}, \ldots, n_{s}}^{\star 2}=$ $\mathcal{H}_{n_{1}, \ldots, n_{s}}^{\star 1}\left[X^{\prime \prime \prime}\right]+e_{3}$, where $e_{3}=\left\{\left(n_{2}-1, n_{2}-1,1, \ldots, 1\right),\left(n_{2}-1, n_{2}-1, n_{3}, \ldots, n_{s-2}, n_{s}\right)\right.$, $\left.\left(n_{1}, \ldots, n_{s-2}, n_{s}\right)\right\}$. By the discussion in Theorems 3.4 3.6. we have the following result.

Theorem 3.7 If $n_{1}-1, n_{s}+1 \in S$, then $\mathcal{H}_{n_{1}, \ldots, n_{s}}^{\star 2}$ is a one-realization of $S$.

Combining Theorem 3.1, Lemma 3.2, Theorems 3.4 3.7, we complete the proof of Theorem 1.2.

\section{Acknowledgment}

The research is supported by NSF of Shandong Province (No. ZR2009AM013), NCET08-0052, NSF of China (10871027) and the Fundamental Research Funds for the Central Universities of China. 


\section{References}

[1] C. Bujtás, Zs. Tuza, Uniform mixed hypergraphs: the possible numbers of colors, Graphs and Combin. 24 (2008), 1-12.

[2] K. Diao, P. Zhao and H. Zhou, About the upper chromatic number of a cohypergraph, Discrete Math. 220 (2000), 67-73.

[3] K. Diao, G. Liu, D. Rautenbach and P. Zhao, A note on the least number of edges of 3-uniform hypergraphs with upper chromatic number 2, Discrete Math. 306 (2006), 670-672.

[4] T. Jiang, D. Mubayi, Zs. Tuza, V. Voloshin and D. West, The chromatic spectrum of mixed hypergraphs, Graphs and Combin. 18 (2002), 309-318.

[5] D. Kobler and A. Kündgen, Gaps in the chromatic spectrum of face-constrained plane graphs, Electronic J. Combin. 8 (2001), $\sharp$ N3.

[6] D. Král, On feasible sets of mixed hypergraphs, Electronic J. Combin. 11 (2004), $\sharp R 19$.

[7] A. Kündgen, E. Mendelsohn and V. Voloshin, Coloring of planar mixed hypergraphs, Electronic J. Combin. 7 (2000), $\sharp$ R60.

[8] Zs. Tuza and V. Voloshin, Problems and results on colorings of mixed hypergraphs, Horizons of Combinatorics, Bolyai Society Mathematical Studies 17, SpringerVerlag, 2008, pp. 235-255.

[9] V. Voloshin, On the upper chromatic number of a hypergraph, Australasian $J$. Combin. 11 (1995), 25-45.

[10] V. Voloshin, Coloring Mixed Hypergraphs: Theory, Algorithms and Applications, AMS, Providence, 2002.

[11] V. Voloshin, Introduction to Graph and Hypergraphs Theory, Nova Scinece Publishers, Inc., New York, 2009.

[12] P. Zhao, K. Diao and K. Wang, The chromatic spectrum of 3-uniform bihypergraphs, Discrete Math. 311(2011), 2650-2656.

[13] P. Zhao, K. Diao and K. Wang, The smallest one-realization of a given set, Electronic J. Combin. 19 (2012), $\sharp \mathrm{P} 19$.

[14] P. Zhao, K. Diao, R. Chang and K. Wang, The smallest one-realization of a given set II, arXiv:1111.1368v1 [math.CO]. 